\title{
CRISPR Interference Efficiently Silences Latent and Lytic Viral Genes in Kaposi's Sarcoma-Associated Herpesvirus-Infected Cells
}

\author{
Kevin Brackett ${ }^{1}$, Ameera Mungale ${ }^{1}$, Mary Lopez-Isidro ${ }^{1}$, Duncan A. Proctor ${ }^{1}$, Guillermo Najarro ${ }^{1}$ \\ and Carolina Arias 1,2,3,*iD \\ 1 Department of Molecular, Cellular, and Developmental Biology, University of California, \\ Santa Barbara, CA 93106, USA; kevinlbrackett@ucsb.edu (K.B.); ameeramungale@umail.ucsb.edu (A.M.); \\ mlopezisidro@umail.ucsb.edu (M.L.-I.); duncan@ucsb.edu (D.A.P.); gnajarro@ucsb.edu (G.N.) \\ 2 Neuroscience Research Institute, University of California, Santa Barbara, CA 93106, USA \\ 3 Center for Stem Cell Biology and Engineering, University of California, Santa Barbara, CA 93106, USA \\ * Correspondence: carolinaarias@ucsb.edu
}

check for updates

Citation: Brackett, K.; Mungale, A.; Lopez-Isidro, M.; Proctor, D.A.; Najarro, G.; Arias, C. CRISPR Interference Efficiently Silences Latent and Lytic Viral Genes in Kaposi's Sarcoma-Associated Herpesvirus-Infected Cells. Viruses 2021, 13, 783. https://doi.org/ $10.3390 /$ v13050783

Academic Editor: Jennifer Corcoran

Received: 7 April 2021

Accepted: 22 April 2021

Published: 28 April 2021

Publisher's Note: MDPI stays neutral with regard to jurisdictional claims in published maps and institutional affiliations.

Copyright: (c) 2021 by the authors. Licensee MDPI, Basel, Switzerland. This article is an open access article distributed under the terms and conditions of the Creative Commons Attribution (CC BY) license (https:/ / creativecommons.org/licenses/by/ $4.0 /)$.

\begin{abstract}
Uncovering viral gene functions requires the modulation of gene expression through overexpression or loss-of-function. CRISPR interference (CRISPRi), a modification of the CRISPRCas9 gene editing technology, allows specific and efficient transcriptional silencing without genetic ablation. CRISPRi has been used to silence eukaryotic and prokaryotic genes at the single-gene and genome-wide levels. Here, we report the use of CRISPRi to silence latent and lytic viral genes, with an efficiency of $\sim 80-90 \%$, in epithelial and B-cells carrying multiple copies of the Kaposi's sarcoma-associated herpesvirus (KSHV) genome. Our results validate CRISPRi for the analysis of KSHV viral elements, providing a functional genomics tool for studying virus-host interactions.
\end{abstract}

Keywords: KSHV; CRISPR-interference; dCas9-KRAB; Kaposi's sarcoma-associated herpesvirus; gene expression; gene silencing

\section{Introduction}

Kaposi's sarcoma-associated herpesvirus (KSHV) is an oncogenic gammaherpesvirus associated with the development of Kaposi's sarcoma (KS) and two lymphoproliferative disorders, primary effusion lymphoma (PEL) and multicentric Castleman's disease (MCD), in immunocompromised patients [1]. KSHV is endemic to Sub-Saharan Africa, where it remains a prevalent medical problem, with $\sim 40$ thousand cases of KS each year, accounting for approximately $20 \%$ of all cancers in the region (fifth most common cancer) [2,3]. In the United States, 1 in 200 transplant patients develop pathologies associated with KSHV reactivation, leading to transplant rejection or death [4].

KSHV has a large, circular dsDNA genome of $160-170 \mathrm{~Kb}$, which encodes over 90 open reading frames (ORFs), more than two dozen short ORFs and upstream ORFs, circular RNAs, several long noncoding RNAs (ncRNAs), and 25 micro RNAs [5,6]. Functional genomics studies have revealed the role of several viral ORFs and ncRNAs in immunomodulation [7], oncogenesis [3,8], and the basic biology of the abduction of the cellular machinery by the virus [9-13]. Nevertheless, several viral elements' biological roles, including long ncRNAs and alternative transcripts, remain unclear. The generation of loss-of-function mutants is the most effective strategy for the functional characterization of viral genes. The construction of KSHV bacterial artificial chromosome (BAC) clones and recombineering techniques have propelled the systematic analysis of KSHV gene functions [14,15]. However, recombineering KSHV BACs remains a time-consuming and often challenging endeavor, typically restricted to one or a few genes.

Post-transcriptional gene silencing and genome editing through RNA interference (RNAi) and CRISPR-Cas9, respectively, have expanded the functional genomics toolkit to study KSHV genes' function and virus-host interactions [9,16-20]. RNAi using small 
interfering RNAs (siRNAs) and short hairpin RNAs (shRNAs) has been useful for depleting many KSHV transcripts. However, RNAi has inherent limitations, including off-target effects, the competition with endogenous substrates for the cell's RNAi machinery, and their inability to target nuclear transcripts [21-23]. CRISPR editing overcomes these limitations and has been recently used to generate single viral gene knockouts or as part of genetic screens in KSHV-infected cells [16-18,24]. Nevertheless, CRISPR editing is encumbered by the viral genome multiplicity naturally observed in KSHV-infected cells, ranging from a handful to over 100 viral genomes per cell [25]. Consequently, CRISPR editing of viral loci present in an infected cell can be a mammoth undertaking, requiring the screening and selection of single cells carrying mutations in every KSHV episome [24]. Moreover, even under stringent selection conditions, it is still difficult to ensure that every viral genome has been edited to carry the same mutation.

CRISPR interference (CRISPRi) offers an alternative approach. CRISPRi represses gene expression using a catalytically dead Cas9 (dCas9) fused to the transcriptional repressor domain Krüppel-associated box (KRAB), recruited to gene promoters or open reading frames by sgRNAs [26,27]. Upon binding to the target DNA sequence, the dCas9-KRAB complex (dC9K) represses transcription initiation or elongation by steric hindrance [26]. Repression is enhanced by the KRAB domain-mediated recruitment of heterochromatin-forming factors, which epigenetically silence the gene [28]. Unlike CRISPR-editing, CRISPRi does not require the generation of individual mutations at single loci to achieve a close to complete loss-of-function phenotypes in mammalian, yeast, and bacterial cells [29-31]. This characteristic makes CRISPRi an ideal technology to carry out functional genomic studies in virus-infected cells where the simultaneous targeting of multiple individual episomes is required for effective viral gene silencing. Here, we report the development of a CRISPRi system to silence viral genes in KSHV-infected epithelial and B-cells. We show that CRISPRi efficiently represses latent and lytic KSHV genes and that it is unfettered by the numerous viral genomes present in infected cells. CRISPRi complements traditional mutagenesis approaches and offers a straightforward and rapid alternative for the interrogation and characterization of KSHV gene functions.

\section{Materials and Methods}

\subsection{Cell Culture}

iSLK.219 and HEK 293METR cells were grown in Dulbecco's modified Eagle medium (DMEM; Invitrogen, Carlsbad, CA, USA) supplemented with 10\% FBS, $200 \mathrm{uM}$ of Lglutamine, and $100 \mathrm{U} / \mathrm{mL}$ of penicillin and streptomycin. iSLK-219 cells were maintained in $10 \mu \mathrm{g} / \mathrm{mL}$ of puromycin (Invivogen, San Diego, CA, USA). BCBL-1 were grown in RPMI 1640 medium (Invitrogen, Carlsbad, CA, USA) supplemented with 10\% fetal bovine serum (FBS; Invitrogen, Carlsbad, CA, USA), $200 \mu \mathrm{M}$ of L-glutamine, and $100 \mathrm{U} / \mathrm{mL}$ of penicillin/streptomycin. Exogenous RTA expression was induced in iSLK-219 cells by treatment with $1 \mu \mathrm{g} / \mathrm{mL}$ of doxycycline (Fisher Scientific, Waltham, MA, USA).

\subsection{Generation of iSLK-219- $d C 9 K$ and $B C B L-1-d C 9 K$}

VSV-G pseudotyped lentiviruses for the generation of cell lines expressing dCas9BFP-KRAB (IGI-p0165 generous gift of the Innovative Genomics Institute, Berkeley, CA 94704, USA) were produced using standard protocols in HEK293-METR cells [32]. Lentiviral supernatants were collected in virus collection medium (DMEM containing $4.5 \mathrm{~g} / \mathrm{L}$ glucose supplemented with 4-10\% FBS, 15 mM HEPES, L-glutamine, sodium pyruvate and penicillin/streptomycin) and concentrated using regenerated cellulose centrifugal filter units with a $100 \mathrm{k}$ MW cut-off (Amicon Ultracel $100 \mathrm{k} / 3000 \mathrm{rpm} / 10 \mathrm{~min}$ ). The concentrated lentiviral supernatant was used to infect iSLK-219 or BCBL-1 cells by spinoculation ( $2000 \mathrm{rpm} / 2 \mathrm{~h} / \mathrm{RT}$ ) in 6 well plates. The cells were allowed to recover for $24 \mathrm{~h}$ and were selected with blasticidin $(10 \mu \mathrm{g} / \mathrm{mL})$ for 10 days. The cells were then sorted for BFP expression in the FACSAria II sorter (BD). 


\section{3. sgRNA Design and Transduction}

sgRNAs for CRISPRi of KSHV (accession number GQ994935.1) were designed as described by Horlbeck et al. [33] (Table S1). Synthetic DNA segments encoding the sgRNAs were cloned into the pHR-SFFV-dCas9-BFP-KRAB (Addgene 46911) at BstXI and XhoI, and the clones were confirmed by sanger sequencing. Lentiviral production and transduction were performed as described above. After transduction, BCBL-1 cells were maintained in $1 \mu \mathrm{g} / \mathrm{mL}$ of puromycin for 10 days prior to the selection of BFP+/sgRNA+ by FACS in a Sony SH800 instrument. iSLK-219 cells were selected for BFP+/sgRNA+ expression by FACS in a Sony SH800 instrument (see Tables S1 and S2 for sgRNA sequences and genomic coordinates).

\subsection{Immunoblotting and Antibodies}

Cells were washed and collected in $1 \times$ sample buffer $(62.5 \mathrm{mM}$ Tris- $\mathrm{HCl}(\mathrm{pH} 6.8)$, $2 \%$ sodium dodecyl sulfate (SDS), $10 \%$ glycerol, $0.7 \mathrm{M} \beta$-mercaptoethanol). Cell lysates were fractionated by SDS-PAGE and transferred onto nitrocellulose membranes. Immunoblots were incubated with primary antibodies overnight at $4{ }^{\circ} \mathrm{C}$ and immunoreactive bands were detected with HRP-conjugated secondary antibodies by enhanced chemiluminescence (ThermoFisher, Waltham, MA, USA) according to the manufacturer's recommendations. All antibodies were used at a 1:1000 dilution in 3\% BSA/1× TBST, unless indicated. Antibodies: ORF57 (SBCT sc-135746), ORF59 (Advanced Biotechnology 13-211100), PERK (Cell Signaling 5683), bActin (1:30,000, Sigma Aldrich, St. Louis, MO, USA), GAPDH (1:20,000 Abcam, Cambridge, UK), Vinculin (Cell Signaling 4650), K8.1 (mAb clone 19B4) [34]. The LANA rabbit polyclonal antibody was raised against a synthetic peptide from the acidic domain of LANA (Polson and Ganem, unpublished).

\subsection{Immunofluorescence}

Cells were fixed in $4 \%$ paraformaldehyde (PFA) at room temperature for ten minutes, or in $90 \%$ ice-cold methanol as indicated. Fixed cells were washed with $1 \times$ PBS and blocked in 3\% BSA $/ 1 \times$ PBS $/ 0.1 \%$ Triton, at room temperature for $1 \mathrm{~h}$. Cells were incubated with 1:1000 Rabbit anti-LANA antibody overnight at $4{ }^{\circ} \mathrm{C}$. Cells were washed with PBS- $0.1 \%$ Triton (PBST) and incubated with a secondary antibody (anti-Rabbit Alexa 488, 1:2500) and DAPI, for $1 \mathrm{~h}$ at room temperature. Cells were washed with PBST and then imaged using the LEICA SP8 confocal microscope.

\subsection{Quantitative Reverse Transcription PCR}

Cells were lysed with RLT Lysis Buffer and RNA was extracted using an RNeasy Mini Kit (Qiagen, Hilden, Germany). cDNA synthesis was performed using iScript cDNA Synthesis Kit (Bio-Rad) following the manufacturer's recommendations. Quantitative polymerase chain reaction (qPCR) was performed in triplicate using the $2 \times \mathrm{SYBR}$ green mix (ThermoFisher, Waltham, MA, USA). Primer sequences for ATF6 Fwd-5' CCTGCTGTTACCAGCTACCAC-3' ${ }^{\prime}$, Rev-5'-CCAAAGAAGGTGTTGGTTTGA-3'; primer sequences for $28 \mathrm{~S}$ Fwd-5'-AAACTCTGGTGGAGGTCCGT-3', Rev-5'-CTTACCAAAAGTG GCCCACTA-3'.

\subsection{Virus Tittering}

The viral titer was determined by collecting lytic cells' supernatants that were clarified, filtered with a $0.45 \mathrm{um}$ syringe filter, and diluted before the spinoculation $(2000 \mathrm{rpm} / 2 \mathrm{~h} /$ Room temp) of uninfected iSLK in 6 well plates. Cells were incubated for $48 \mathrm{~h}$, trypsinized and collected for flow cytometry in a Sony SH800 instrument. The percentage of cells expressing EGFP was determined by flow cytometry and used to calculate the number of fluorescence forming units (ffus) in each sample. 


\section{Results}

\subsection{CRISPRi Represses Viral Genes in KSHV Infected Cells}

To silence viral genes using CRISPRi, we engineered two KSHV latently infected cell lines, iSLK-219 and BCBL-1, to constitutively and stably express dCas9-KRAB (dC9K) (Figure 1A). The epithelial cell line iSLK-219, and the B-cell line BCBL-1 are robust, commonly used KSHV infection models $[35,36]$. iSLK-219s are latently infected with the recombinant KSHV.219 strain, which encodes constitutive (EGFP) and lytic-reactivation-inducible (RFP) reporters [37]. These cells also harbor a doxycycline (Dox) inducible transgene encoding the viral transcription factor RTA (replication and transcriptional activator), which acts as the master switch for the transition from the latent to the lytic stages of infection [38]. BCBL-1 cells are derived from a PEL patient and are naturally infected with wild-type KSHV [35]. To generate CRISPRi KSHV-infected cells, we transduced iSLK-219 and BCBL-1 cells with lentiviruses encoding dC9K fused to a blue fluorescent protein (BFP). Initially, we attempted to establish iSLK-219 cell lines that stably express dC9K but noted that the transgene was lost after transduction (data not shown). We used a lentiviral vector with a blasticidin resistance marker to overcome this limitation and maintained the transgene for several generations under blasticidin selection. Finally, we selected pseudoclonal cell populations of high dC9K expression by fluorescence-activated cell sorting (FACS) and maintained the sorted cells under blasticidin selection (Figure 1A).

A
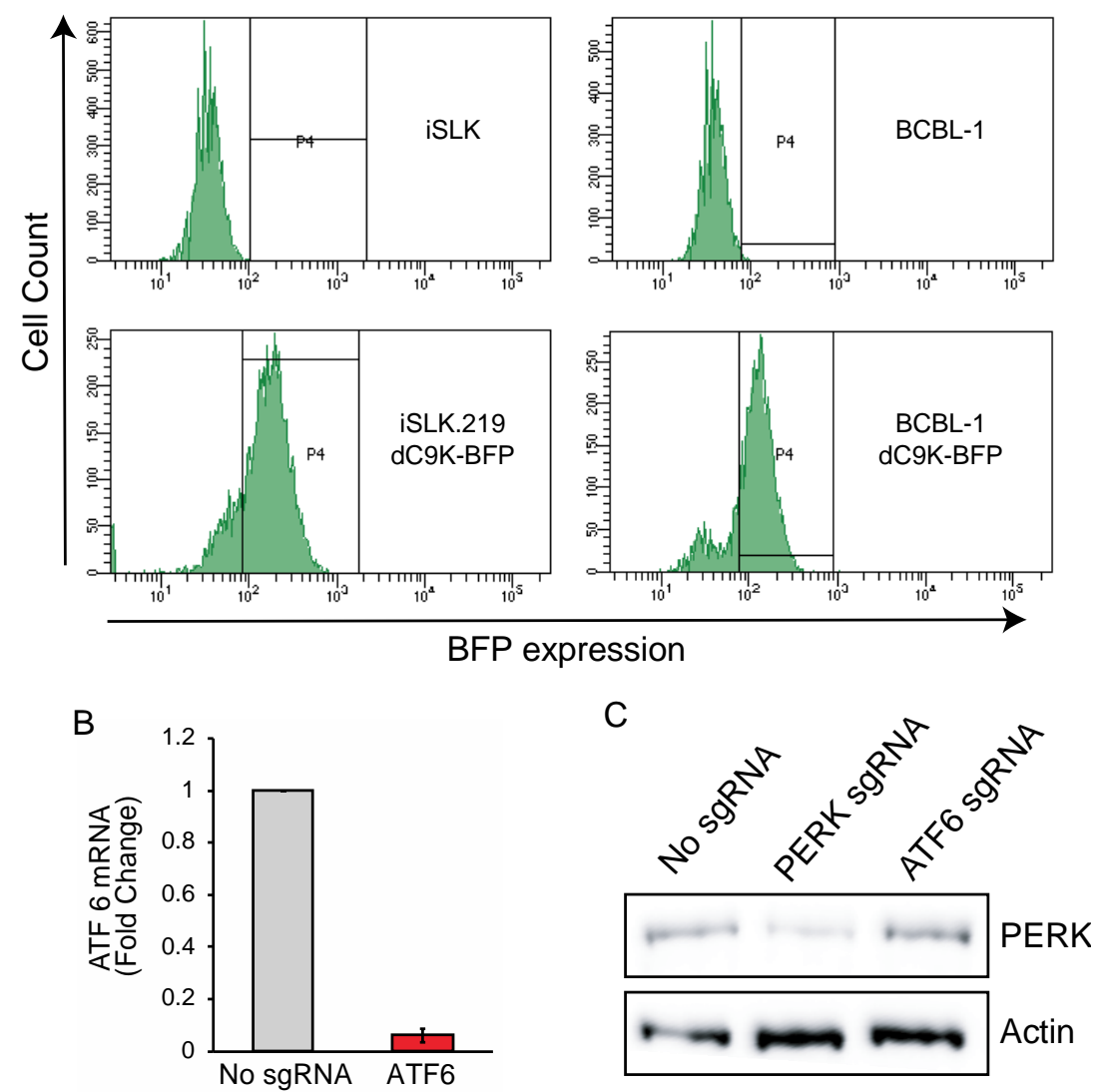

Figure 1. Generation of iSLK-219-dC9K and BCBL-1-dC9K cell lines. (A) FACS traces showing mean fluorescence intensity (MFI) of iSLK-219 and BCBL-1 cells transduced with a lentiviral vector expressing dCas9-KRAB-BFP-Blasticidin, selected with blasticidin for 10 days and sorted by FACS for BFP+ expression (P4 gate) (B) RT-qPCR showing silencing of ATF6 in BCBL-1 cells. BCBL-1-dC9K cells were transduced with an ATF6 sgRNA targeting vector and selected with puromycin for 10 days. $28 \mathrm{~S}$ rRNA was used as a loading control. Error bars: standard deviation (C) immunoblot showing silencing of PERK 10 days after iSLK-219 cells were transduced with a PERK sgRNA targeting vector and selected by FACS (BFP+). 
To test whether the CRISPRi system was functional in the cell lines mentioned above, we transduced them with sgRNAs targeting the promoters of the endogenous genes PERK and ATF6, which encode ubiquitously expressed endoplasmic reticulum stress sensor proteins (Table S1) [39]. The lentiviral vector encoding the sgRNAs also encodes BFP and a puromycin selection marker, allowing the pharmacological and FACS-based selection of positive transductants. We selected BCBL-1-dC9K cells expressing the ATF6 targeting sgRNAs by treating them with puromycin. Next, we measured the expression levels of ATF6 by RT-qPCR and observed robust repression ( $>90 \%$ ) of the corresponding mRNA in cells expressing the targeting sgRNA (Figure 1B). As the KSHV.219 recombinant virus present in iSLK-219-dC9K has a puromycin resistance marker, we selected $\mathrm{BFP}^{+}$cells expressing the PERK targeting sgRNA by FACS. The expression of BFP is more efficient from the sgRNA vector than from the dCas9-KRAB vector, thus allowing for the selection of sgRNA transduced cells based on $\mathrm{BFP}^{+}$status. We evaluated the efficiency of PERK silencing by CRISPRi in the iSLK-219-dC9K cell by immunoblot and observed a substantial reduction in PERK protein levels (Figure 1C). Together, these results show that, expectedly, CRISPRi efficiently silences host gene promoters in KSHV-infected iSLK.219 and BCBL-1 cells (Figure 1B,C).

Next, we evaluated the efficiency of CRISPRi for silencing viral genes. We reasoned that the multiple copies of KSHV episomes could reduce the inhibitory effect of the CRISPRi machinery on viral genes by promoter competition and stoichiometry. To test whether CRISPRi can overcome the constraint imposed by viral gene expression derived from multiple transcriptionally active episomes, we transduced iSLK-219 cells with sgRNAs targeting the transcription start site (TSS) of the EGFP reporter gene encoded in the KSHV.219 genome (Figure 2A; Table S1). We chose EGFP to evaluate CRISPRi silencing because it is ubiquitously expressed in latently infected cells under the control of a constitutive EF-1 promoter, and it allows for the straightforward monitoring of changes in protein levels. Using flow cytometry, we detected sgRNA/BFP expression in greater than $80 \%$ of the transduced cells (Figure 2B), and we evaluated the levels of EGFP expression in unselected/unsorted cells. We found that the iSLK-219-dC9K cells expressing the targeting sgRNA $\left(\mathrm{BFP}^{+}\right)$have lower levels of EGFP when compared to non-transduced $\left(\mathrm{BFP}^{-}\right)$cells (Figure 2B). To confirm that the reduction in EGFP levels in sgRNA transduced cells was specific and not the result of episome loss, we immunostained for the latency-associated nuclear antigen (LANA), a viral protein required for the maintenance of latent infection. In these experiments, we observed that transduced cells showed low levels of EGFP, as well as the characteristic LANA punctate pattern that is associated with latent KSHV infection (Figure 2C). This observation indicates that the viral genome is still present and that LANA expression was unaltered after EGFP silencing. Together, these results demonstrate that CRISPRi is effective for silencing viral genes and is not hampered by the 30-100 copies of the KSHV genome present in infected cells.

\subsection{CRISPRi Represses Latent and Lytic KSHV Genes and Curtails Infectivity}

The viral cycle is divided into two main phases: latency, a persistent and dormant state with low viral gene expression, and the lytic cycle, a transcriptionally and translationally active state and the productive stage of infection. Having determined that CRISPRi can effectively repress viral-encoded genes (EGFP, Figure 2B), we next examined whether CRISPRi is equally effective at silencing latent and lytic genes. First, we explored CRISPRi silencing of a KSHV latent gene. To this end, we chose to silence LANA, a key mediator of latent viral replication that tethers the viral episome to host chromosomes by bridging the interaction between the KSHV genome and host chromatin [40-42]. To test whether CRISPRi repressed LANA, we transduced iSLK-219-dC9K cells with sgRNAs targeting the LTc promoter (Table S2, Figure 3A), which is known to be preferentially used for LANA transcription in these cells [6]. Two weeks after transduction, we selected the upper quartile of $\mathrm{BFP}^{+} / \mathrm{sgRNA}^{+}$expressing cells by FACS and evaluated LANA levels in this population. We observed a substantial reduction in LANA expression by immunofluorescence and 
immunoblot analyses ( 90\% expression loss, $p<0.0001)$ (Figure 3B,C) and a concomitant reduction in the expression of the virus-encoded EGFP reporter by flow cytometry in cells transduced with LANA-targeting sgRNAs (Figure 3D). These results indicate that CRISPRi loss-of-function of LANA results in the loss of viral episomes and agree with recent observations showing that CRISPR-Cas9-mediated mutagenesis of LANA results in episome loss and latency disruption [18]. Together, these results indicate that CRISPRi can mediate the efficient knockdown of KSHV latent genes.

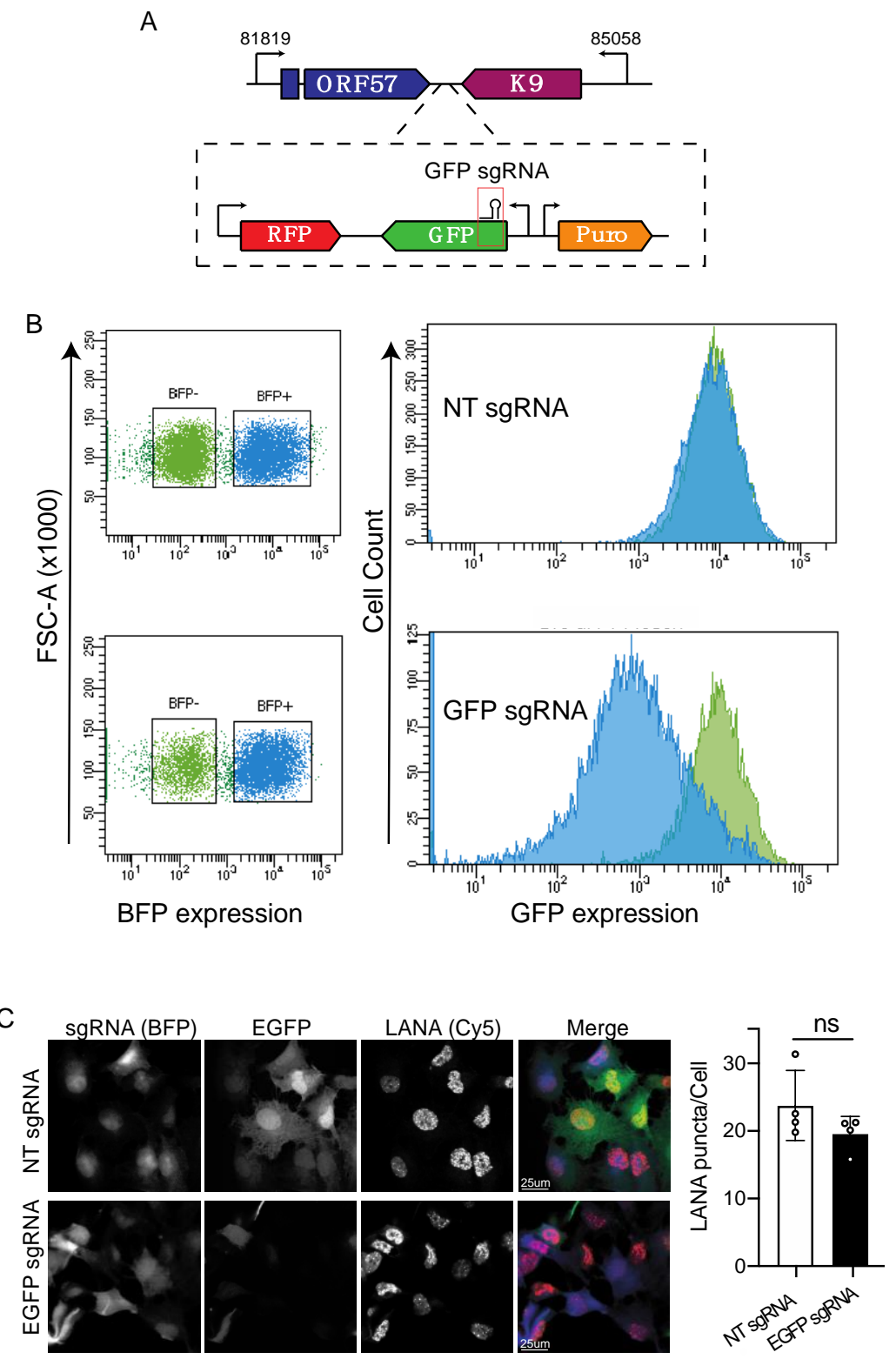

Figure 2. CRISPRi silencing of KSHV-encoded EGFP. (A) Region of the viral genome encoding EGFP and position of the sgRNA. (B) Flow cytometry analysis (MFI) of iSLK-219-dC9K cells transduced with sgRNAs targeting EGFP or non-targeting (NT) sgRNAs. Note the downregulation of EGFP expression in cells transduced with the targeting sgRNA. (C) Immunofluorescence and confocal microscopy analyses of paraformaldehyde-fixed, BFP expressing cells (sgRNA), EGFP and LANA in iSLK-219-dC9K cells transduced with sgRNAs targeting EGFP or non-targeting (NT) sgRNAs. Quantification of LANA puncta/Cell in four independent fields ( $t$-Test, ns $p=0.15)$. 
A

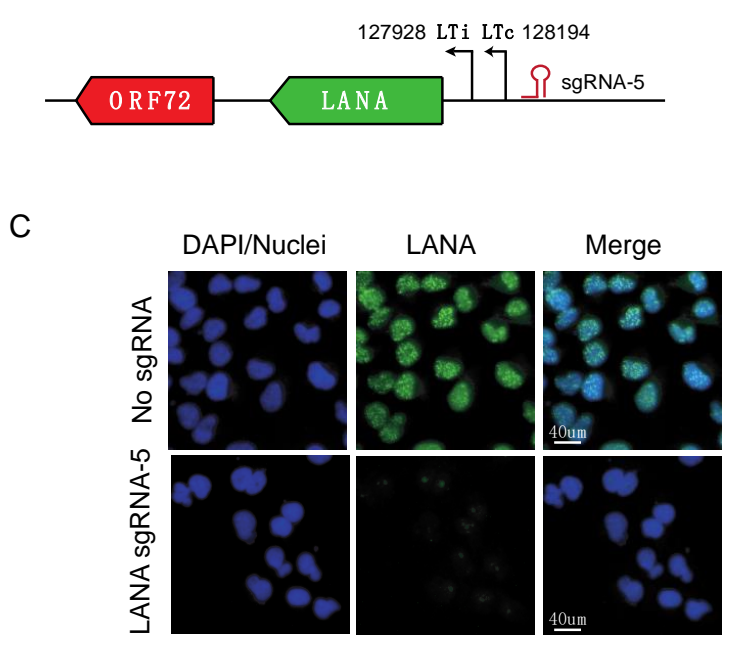

B

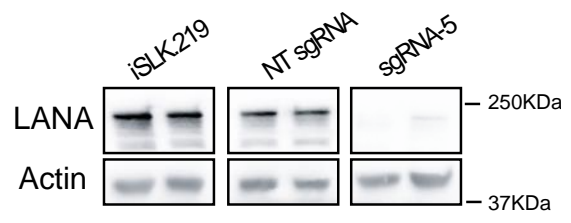

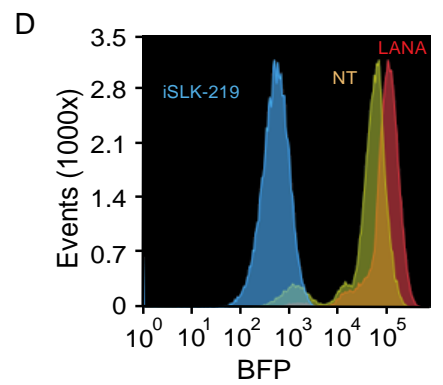

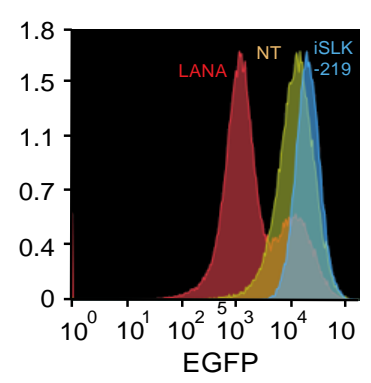

Figure 3. CRISPRi silencing of latent genes in iSLK-219 cells. (A) Region of the viral genome encoding LANA (ORF73) and position of the sgRNA. (B) Immunoblot analysis of LANA in iSLK-219 cells, and iSLK-219-dC9K cells transduced with a non-targeting (NT) sgRNA or a LANA specific sgRNA. Image representative of three biological replicates. Bars: quantification of the data. (one-way ANOVA, **** $p<0.0001)$. Error bars: standard deviation, ns, not significant. (C) Immunofluorescence analyses of LANA in methanol-fixed iSLK-219-dC9K cells transduced with a LANA specific sgRNA. Image representative of three biological replicates. (D) Flow cytometry analyses (MFI) of iSLK-219 (blue trace), or iSLK-219-dC9K cells transduced with a non-targeting (NT) sgRNA (yellow trace) or a LANA specific sgRNA (red trace). Note the downregulation of EGFP expression in cells where LANA has been silenced. Image representative of two biological replicates.

Next, we determined the ability of CRISPRi to silence lytic genes. KSHV lytic gene expression is classified into three stages based on their kinetics: immediate early, in which the first genes expressed upon entry to the lytic cycle are observed; delayed early, characterized by the expression of genes before DNA replication; and late, in which genes are expressed after DNA replication [43]. We first examined the CRISPRi-mediated silencing of the immediate early lytic gene ORF57, also known as mRNA transcript accumulation (MTA). ORF57 plays a crucial role in viral gene expression by enhancing RNA stability and splicing and promoting protein synthesis. Consequently, ORF57 deletion, silencing, or inactivation reduces viral reactivation and replication $[44,45]$. To silence ORF57, we transduced iSLK-219-dC9K cells with a sgRNA targeting the promoter at the annotated TSS ( -3 to $+15 \mathrm{bp}$ ) (Table S2, Figure $4 \mathrm{~A})$. Ten days after transduction, we selected the upper quartile of cells based on $\mathrm{BFP}^{+} /$sgRNA expression levels by FACS. We prompted entry into the lytic cycle in this population by exogenous RTA expression induced by doxycycline treatment [36]. We evaluated the expression of ORF57 at $0 \mathrm{~h}$ (latent), $24 \mathrm{~h}$ (early lytic), and $48 \mathrm{~h}$ (late lytic) post-reactivation and found significant downregulation ( $90 \%$ knockdown, 
$p<0.001)$ at $24 \mathrm{~h}$ post-reactivation. The potency of CRISPRi inhibition was lower yet statistically significant at $48 \mathrm{~h}$ post-reactivation $(\sim 50 \%$ knockdown, $p=0.025)$, possibly due to an increase in the number of viral genomes following viral DNA replication and dilution of the repressive effect of dC9K. The silencing of ORF57, even when partial at $48 \mathrm{~h}$, caused a substantial reduction in the expression of viral lytic genes, including K8.1, a canonical lytic marker $(\sim 86 \%$ reduction, $p<0.001)$ (Figure $4 \mathrm{~B})$, and a drop in viral replication $(80-85 \%$ reduction in viral titers, $p<0.0001$ ) (Figure $4 \mathrm{C}$ ), without any measurable impact on latent gene expression (4D). Together, these results indicate that CRISPRi can efficiently silence immediate-early lytic KSHV genes.

A
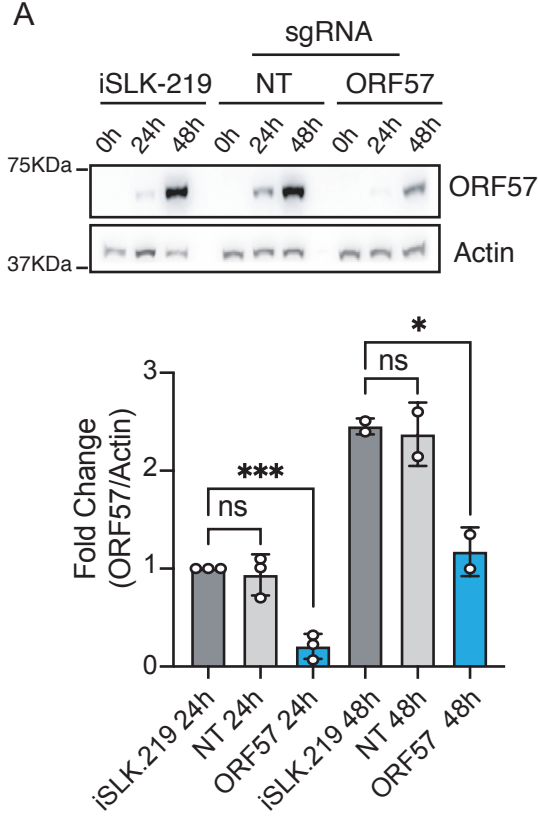

B
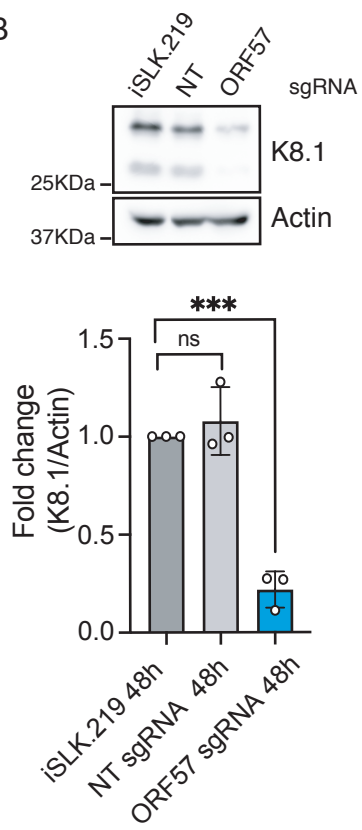

C

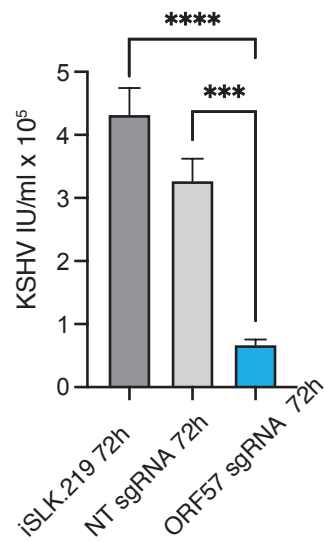

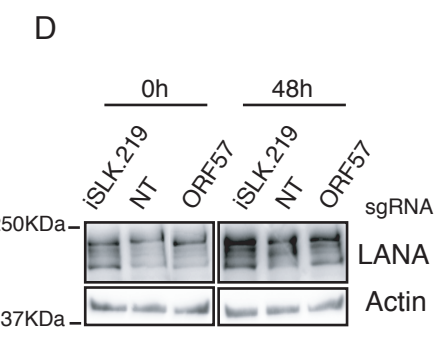

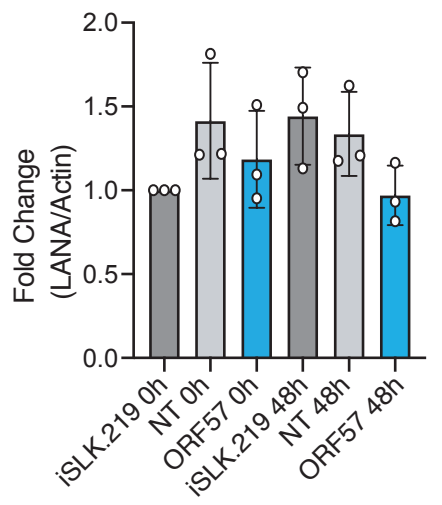

Figure 4. CRISPRi silencing of immediate-early genes in iSLK-219 cells. Immunoblot analysis of (A) ORF57 (one-way ANOVA, $24 \mathrm{~h} * * * *<0.001,48 \mathrm{~h} * p$ value 0.025 ), (B) K8.1 (one-way ANOVA, $48 \mathrm{~h} * * *<0.001$ ) and (D) LANA (Kruskal-Wallis test, $0.99<p<0.3$ ) in latent and lytic (24 and $48 \mathrm{~h}$ post-reactivation) iSLK-219 cells, and iSLK-219-dC9K cells transduced with a non-targeting (NT) sgRNA or an ORF57-specific sgRNA. Image representative of three biological replicates. Bars: quantification of the data. Error bars: standard deviation, ns, not significant. (C) Quantification of viral titers (IU, infectious units) in the filtered supernatant of iSLK-219 or iSLK-219-dC9K cells transduced with a non-targeting (NT) sgRNA or an ORF57-specific sgRNA, collected at $72 \mathrm{~h}$ postreactivation. Error bars: standard deviation. (one-way ANOVA, ${ }^{* * * *} p<0.001,{ }^{* * *} p<0.001$ ).

Motivated by these findings, we next investigated whether CRISPRi can repress the transcription of KSHV delayed-early lytic genes. To this end, we focused on the promoter of 
ORF59, a viral processivity factor that coordinates the translocation of the viral polymerase (ORF9) to the nucleus. The ablation of ORF59 disrupts DNA replication and impedes virion production [46-48]. We targeted the promoter of ORF59 with two sgRNAs in the proximal region of the TSS ( $+24 \mathrm{bp}$ from TSS on the template strand and $+14 \mathrm{bp}$ from TSS on the coding strand) (Table S2, Figure 5A). Ten days after transduction, we selected the upper quartile of cells expressing $\mathrm{BFP}^{+} / \mathrm{sgRNA}^{+}$by FACS and induced entry to the lytic cycle by doxycycline-driven exogenous expression of RTA. After $96 \mathrm{~h}$ had passed following reactivation, we evaluated the expression of ORF59 by immunoblot and found a substantial downregulation of ORF59 ( $>90 \%$ reduction, $p<0.0001)$ (Figure 5B). As anticipated, and due to the critical function of ORF59 during the lytic cycle, the silencing of ORF59 resulted in the loss of late lytic proteins, including K8.1 (73-75\% reduction, $p<0.0001)$ (Figure 5B), and a concomitant decrease in virus replication (80-90\% reduction in titers) (Figure 5C). Our results show that CRISPRi efficiently represses delayed early lytic genes.

A

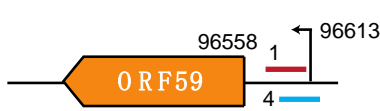

C

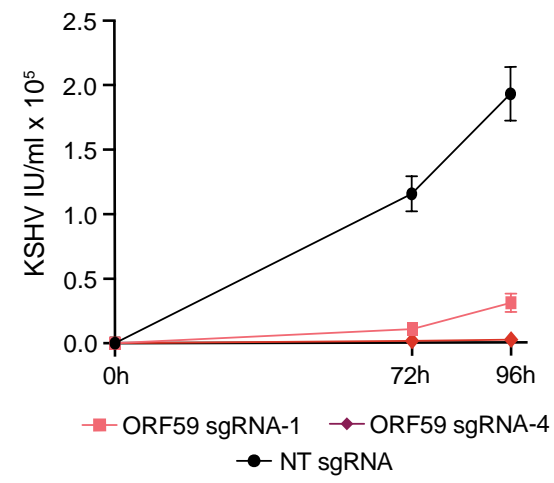

B
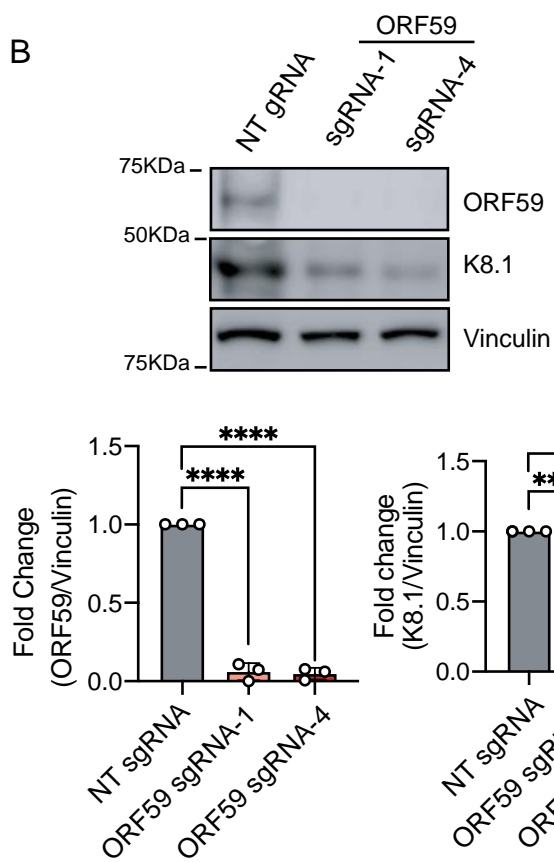

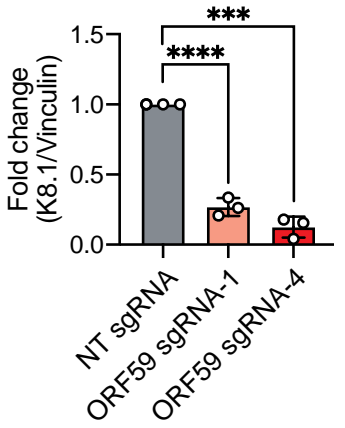

Figure 5. CRISPRi silencing of delayed-early genes in iSLK-219 cells. (A) Region of the viral genome encoding ORF59 and position of the sgRNAs. Immunoblot analysis of (A) ORF59, and (B) K8.1 (in latent and lytic (96 h post-reactivation) in iSLK-219-dC9K cells transduced with a non-targeting (NT) sgRNA or an ORF59-specific sgRNA. Image representative of three biological replicates. Bars: quantification of the data. Error bars: standard deviation (one-way ANOVA, ${ }^{* * * *} p<0.001,{ }^{* * *} p<0.001$ ). (C) Quantification of viral titers (IU, infectious units) in the filtered supernatant of iSLK-219-dC9K cells transduced with a non-targeting (NT) sgRNA or ORF59-specific sgRNAs, collected at 72 and $96 \mathrm{~h}$ post-reactivation. Error bars: standard deviation.

\subsection{CRISPRi Represses Viral Genes in PEL-Derived Cells}

The results obtained in iSLK-219-dC9K cells (Figures 2-5) indicated that CRISPRi silences KSHV genes belonging to different kinetic classes. Since the host cell type can significantly affect the choice of viral promoters and the regulation of viral gene expression in KSHV-infected cell lines [6], we sought to determine the efficiency of CRISPRi in BCBL-1 cells. To this end, we transduced BCBL-1-dC9K cells with sgRNAs targeting the LANA promoter (Table S2). BCBL-1 cells regulate LANA expression through two different promoters LTc and LTi [6,49]. We noted no gene expression changes ten days after transduction when we used the same sgRNA that we successfully used in iSLK-219-dC9K cells, sgRNA-LANA-5 (Figure 6B). Neither did we observe any measurable changes in gene expression when we targeted several other sgRNAs to different regions around the 
LANA LTc promoter (Figure 6B). We reasoned that an alternative promoter choice may compensate for LANA expression in these conditions, so we simultaneously targeted both LANA promoters in BCBL-1-dC9K cells with two sgRNAs complementary to the TSS at the LTc and the LTi promoters (Figure 6A). Using this approach, we observed the significant repression of LANA expression (50-80\% reduction, $p<0.001-0.01$ ) in BCBL-1 cells (Figure 6B,C). We achieved the best silencing when we positioned the sgRNAs immediately downstream of the LTc $(17+$ to $27+\mathrm{bp})$ and the LTi $(21+$ to $30+\mathrm{bp})$ LANA promoters. Together, these results demonstrate that CRISPRi effectively silences KSHV genes in different cellular backgrounds.

A

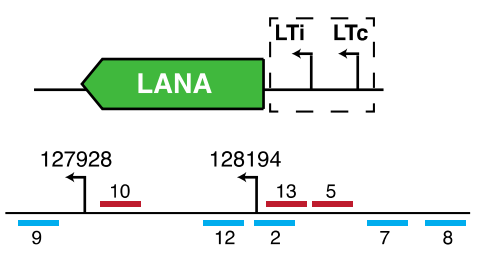

B

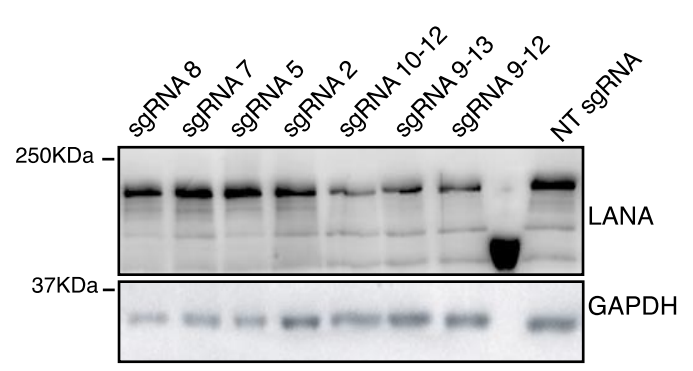

C

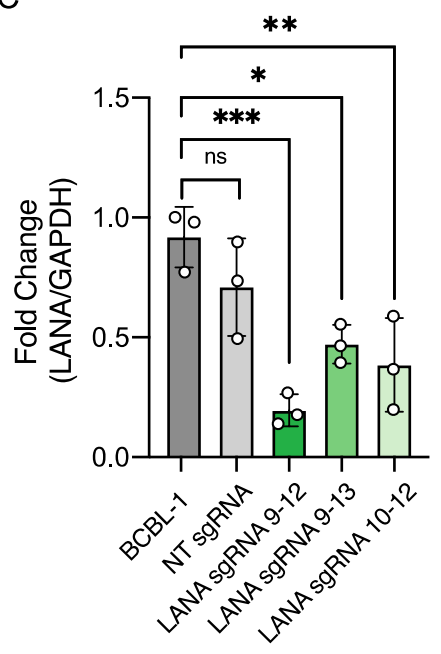

Figure 6. CRISPRi silencing of latent genes in BCBL-1 cells. (A) Region of the viral genome encoding LANA (ORF73) and position of the sgRNAs. (B) Immunoblot analysis of LANA in iSLK-219 cells, and iSLK-219-dC9K cells transduced with a non-targeting (NT) sgRNA or a LANA specific sgRNA. Image representative of three biological replicates. (C) Quantification of the immunoblot data for sgRNAs that silenced LANA. Error bars: standard deviation (one-way ANOVA, ${ }^{* * *} p=0.0004,{ }^{* *} p=0.004$, ${ }^{*} p=0.0124$, ns, not significant).

\section{Discussion}

CRISPR-based technologies have transformed our ability to conduct functional genomics studies. Two modifications of the CRISPR-Cas9 system, CRISPR activation (CRISPRa) and CRISPR interference (CRISPRi), permit manipulating gene expression with unprecedented precision and have opened up new ways for functional gene characterization. CRISPRi has been extensively used in mammalian, plant, and bacterial cells for single-gene and genome-wide transcriptional repression [26,27,29-31]. A recent study by Hein and Weissman highlights the advantages of CRISPRi for the genome-wide study of virus-host interactions, as it allows one to interrogate the role of essential host genes in infection, a feature commonly missed by CRISPR-Cas9 screens, and opens the possibility for interrogating viral gene functions [50]. Here, we show that CRISPRi can be used to repress the expression of latent and lytic KSHV genes, and that these manipulations curtail infectivity in cells in culture. Our results provide proof-of-concept for the use of CRISPRi to characterize viral gene functions.

CRISPRi offers two key advantages over CRISPR-Cas9 gene editing (knockouts) to study viral gene function. First, CRISPRi does not cause dsDNA breaks and it does not rely on the host cell DNA repair machinery, which circumvents the problem of generating different mutations in the multiple copies of the viral genome present in infected cells. Second, CRISPRi can access promoter elements in all episomes, thus obviating the need for targeting every single viral locus to achieve penetrant loss-of-function. Our results show that CRISPRi can be recruited to and repress promoters of latent and lytic genes in the 
multiple KSHV genomes present in these cells. These characteristics make CRISPRi an ideal technology to modulate gene expression in cells infected with herpesviruses.

It is noteworthy that the efficiency of CRISPRi for targeting specific loci seems to depend on the timing of the viral life cycle. For instance, the efficient silencing of ORF57 dwindled as the lytic cycle progressed. It is possible that reduction in the repressive effect of CRISPRi on ORF57 at late timepoints following reactivation is due to the replication of viral DNA and the accumulation of viral genomes, and the saturation of the available dC9K. This notion is supported by our observation of the sustained silencing of ORF59, a delayed-early gene, at $96 \mathrm{~h}$ post reactivation. The ablation of ORF59 function prevents viral DNA replication, and thus, under these conditions, the number of genomic targets for dC9K remain constant. Further optimization of the CRISPRi system in KSHV-infected cells, including the selection of pseudoclonal populations with high levels of dC9K expression, may produce more robust silencing of lytic genes following genome replication. Even without such optimizations, we note that the subdued repression of ORF57 at 48 h postreactivation still resulted in impaired viral replication.

The inhibitory effect of CRISPRi is largely affected by the selection of the targeting sgRNA [51]. CRISPRi is most effective when the sgRNA targets the region $-50 / 150+b p$ from the TSS, with optimum activity in the region between 0/50+ from the TSS [27,51]. Our results corroborate these observations and show that the selection and position of the sgRNA is critical to tune the efficiency of gene repression of target viral genes. The strong silencing of LANA, ORF57, and ORF59 we achieved demonstrates that CRISPRi can approximate viral gene expression levels similar to those seen with loss-of-function approaches. CRISPRi also allows for the interrogation of the function of viral elements that cannot be targeted by RNAi or CRISPR knockouts, including nuclear RNAs and small and long non-coding RNAs, many of which remain uncharacterized in KSHV.

Another strength of CRISPRi for functional genomics studies of virus infected cells is its ability to grade gene expression and protein abundance. As opposed to true knockouts, partial gene loss-of-function can be useful for viral biology studies as it may allow for the bypassing of adaptation effects associated with complete loss-of-function upon gene editing. The careful design of gRNAs could be used to generate hypomorphic alleles by CRISPRi to titrate viral gene expression. Moreover, allelic series based on the expression of different levels of dC9K-sgRNA pairs can be established by selecting pseudoclonal populations. Such populations would allow one to study the effects of viral gene product dosing on infectivity.

The differences we observed in the repression of LANA when using the same sgRNAs in iSLK-219 or BCBL-1 cells may reflect the abundance of viral genomes in epithelial vs. Bcells. BCBL-1 cells have higher numbers of KSHV genomes that could lead to the saturation of available $\mathrm{dC} 9 \mathrm{~K}$ in these cells, resulting in the reduced efficiency of viral gene silencing by CRISPRi. The cell-type-specific regulation of the viral epigenome offers an alternative explanation for the different efficiency of CRISPRi in repressing viral gene expression in either cellular background. Indeed, recent studies on the genome-wide structure and regulation of viral chromatin, and the detailed annotation of the KSHV transcriptome, show that the cellular context has an impact on viral gene expression and transcriptional control. In the particular case of LANA transcription, two promoters, the constitutive LTc and the RTA-responsive LTi, have been identified [52]. A recently detailed annotation of viral transcription start sites in iSLK-219 and BCBL-1 cells show that LTc is used for LANA transcription in both cellular contexts, while LTi is exclusively used in BCBL-1 cells [6]. The factors that determine the selection and use of LTi in different cell lines remain to be determined. These observations underscore the importance of obtaining detailed viral epigenomes and transcriptomes to inform sgRNA design for efficient CRISPRi of viral genes. Another aspect that needs to be carefully considered for viral gene CRISPRi is the compact nature and high density of coding elements of viral genomes, which could increase off-target effects. While CRISPRi has been shown to have minimal off-target effects on cellular genes [26], it will be crucial to determine off-target effects when targeting 
viral genes, particularly those encoded by polycistronic transcripts, and those for which transcription is controlled by bidirectional promoters or promoters with overlapping regulatory elements.

Finally, combining the strengths of CRISPRi with CRISPRa will enable comprehensive functional genomics studies in KSHV and other viruses by providing complementary biological insights. Similar to CRISPRi, CRISPRa targets endogenous promoters to activate genes by inducing their transcription at near-physiological levels, which is often difficult to achieve through ectopic overexpression. A recent report by Elbasani et al. demonstrated the successful activation of the viral transcription factor RTA in KSHV-infected cells, setting the precedent for the use of CRISPRa in viral gene gain-of-function studies [53]. Together, these new technologies will enable the discovery of the characterization of viral features, and guide new biological models in virology.

Supplementary Materials: The following are available online at https:/ /www.mdpi.com/article/10 .3390/v13050783/s1, Table S1: sequence of sgRNAs targeting cellular genes and controls, Table S2: sequences and genomic coordinates of sgRNAs targeting KSHV genes.

Author Contributions: Conceptualization, K.B. and C.A.; methodology, K.B., A.M., M.L.-I., D.A.P., G.N., C.A.; writing—original draft preparation, K.B., C.A.; writing-review and editing, K.B., A.M., M.L.-I., D.A.P., G.N., C.A.; supervision, C.A.; funding acquisition, C.A. All authors have read and agreed to the published version of the manuscript.

Funding: This research was funded by UC Cancer Research Coordinating Committee, grant number CTN-19-586108. G.N. is supported by the National Science Foundation California LSAMP Bridge to the Doctorate (BD) Fellowship Under Grant No. HRD-1701365.

Institutional Review Board Statement: Not Applicable.

Informed Consent Statement: Not Applicable.

Acknowledgments: We thank Luke Gilbert (UCSF), Martin Kampmann (UCSF) and the Innovative Genomics Institute for reagents; Max Horlbeck (UCSF) for the support with the sgRNA design, Monte Radeke (UCSB) for the support with FACS; and Ben Lopez (UCSB) at the NRI-MCDB microscopy facility for the support with microscopy and imaging. We thank Friederike Braig-Karzig and Sabrina Solley for the technical assistance and insightful scientific discussions. We specially thank all members of the Arias and Acosta-Alvear labs for the insightful scientific discussions. We thank Isabel Henley (UCSB) for the critical reading of the manuscript. We thank Diego Acosta-Alvear (UCSB) for the valuable technical advice and critical reading of the manuscript.

Conflicts of Interest: The authors declare no conflict of interest.

\section{References}

1. Dissinger, N.J.; Damania, B. Recent Advances in Understanding Kaposi's Sarcoma-associated Herpesvirus. F1000Research 2016, 5, 740. [CrossRef]

2. International Agency for Research on Cancer. Globocan 2012: Estimated Cancer Incidence, Mortality and Prevalence Worldwide in 2012; World Health Organisation (WHO): Geneva, Switzerland, 2015.

3. Mesri, E.A.; Cesarman, E.; Boshoff, C. Kaposi's Sarcoma and Its Associated Herpesvirus. Nat. Rev. Cancer 2010, 10, 707-719. [CrossRef]

4. What Are the Key Statistics about Kaposi Sarcoma? American Cancer Society: Burlington, VT, USA, 2016.

5. Arias, C.; Weisburd, B.; Stern-Ginossar, N.; Mercier, A.; Madrid, A.S.; Bellare, P.; Holdorf, M.; Weissman, J.S.; Ganem, D. KSHV 2.0: A Comprehensive Annotation of the Kaposi's Sarcoma-Associated Herpesvirus Genome Using Next-Generation Sequencing Reveals Novel Genomic and Functional Features. PLoS Pathog. 2014, 10, e1003847. [CrossRef] [PubMed]

6. Ye, X.; Zhao, Y.; Karijolich, J. The Landscape of Transcription Initiation Across Latent and Lytic KSHV Genomes. PLoS Pathog. 2019, 15, e1007852. [CrossRef]

7. Broussard, G.; Damania, B. KSHV: Immune Modulation and Immunotherapy. Front. Immunol. 2020, 10, 3084. [CrossRef] [PubMed]

8. Cavallin, L.E.; Goldschmidt-Clermont, P.; Mesri, E.A. Molecular and Cellular Mechanisms of KSHV Oncogenesis of Kaposi's Sarcoma Associated with HIV/AIDS. PLoS Pathog. 2014, 10, e1004154. [CrossRef] [PubMed]

9. Chandran, B. Early Events in Kaposi's Sarcoma-Associated Herpesvirus Infection of Target Cells. J. Virol. 2009, 84, 2188-2199. [CrossRef] [PubMed] 
10. Arias, C.; Walsh, D.; Harbell, J.; Wilson, A.C.; Mohr, I. Activation of Host Translational Control Pathways by a Viral Developmental Switch. PLoS Pathog. 2009, 5, e1000334. [CrossRef] [PubMed]

11. MacVeigh-Fierro, D.; Rodriguez, W.; Miles, J.; Muller, M. Stealing the Show: KSHV Hijacks Host RNA Regulatory Pathways to Promote Infection. Viruses 2020, 12, 1024. [CrossRef]

12. Ohsaki, E.; Ueda, K. Interplay Between KSHV and the Host DNA Damage Response. Front. Cell. Infect. Microbiol. 2020, 10, 764. [CrossRef]

13. Kumar, B.; Chandran, B. KSHV Entry and Trafficking in Target Cells-Hijacking of Cell Signal Pathways, Actin and Membrane Dynamics. Viruses 2016, 8, 305. [CrossRef]

14. Zhou, F.C.; Zhang, Y.J.; Deng, J.H.; Wang, X.P.; Pan, H.Y.; Hettler, E.; Gao, S.J. Efficient Infection by a Recombinant Kaposi's Sarcoma-Associated Herpesvirus Cloned in a Bacterial Artificial Chromosome: Application for Genetic Analysis. J. Virol. 2002, 76, 6185-6196. [CrossRef] [PubMed]

15. Brulois, K.F.; Chang, H.; Lee, A.S.Y.; Ensser, A.; Wong, L.Y.; Toth, Z.; Lee, S.H.; Lee, H.-R.; Myoung, J.; Jung, J.U.; et al. Construction and Manipulation of a New Kaposi's Sarcoma-Associated Herpesvirus Bacterial Artificial Chromosome Clone. J. Virol. 2012, 86, 9708-9720. [CrossRef] [PubMed]

16. Liang, Z.; Qin, Z.; Riker, A.I.; Xi, Y. CRISPR/Cas9 Ablating Viral microRNA Promotes Lytic Reactivation of Kaposi's Sarcomaassociated Herpesvirus. Biochem. Biophys. Res. Commun. 2020, 533, 1400-1405. [CrossRef] [PubMed]

17. Gabaev, I.; Williamson, J.C.; Crozier, T.W.; Schulz, T.F.; Lehner, P.J. Quantitative Proteomics Analysis of Lytic KSHV Infection in Human Endothelial Cells Reveals Targets of Viral Immune Modulation. Cell Rep. 2020, 33, 108249. [CrossRef] [PubMed]

18. Tso, F.Y.; West, J.T.; Wood, C. Reduction of Kaposi's Sarcoma-Associated Herpesvirus Latency Using CRISPR-Cas9 To Edit the Latency-Associated Nuclear Antigen Gene. J. Virol. 2019, 93, e02183-18. [CrossRef]

19. Holmes, D.L.; Vogt, D.T.; Lagunoff, M. A CRISPR-Cas9 Screen Identifies Mitochondrial Translation as an Essential Process in Latent KSHV Infection of Human Endothelial Cells. Proc. Natl. Acad. Sci. USA 2020, 117, 28384-28392. [CrossRef] [PubMed]

20. Naik, N.G.; Nguyen, T.H.; Roberts, L.; Fischer, L.T.; Glickman, K.; Golas, G.; Papp, B.; Toth, Z. Epigenetic Factor siRNA Screen during Primary KSHV Infection Identifies Novel Host Restriction Factors for the Lytic Cycle of KSHV. PLoS Pathog. 2020, 16, e1008268. [CrossRef]

21. Jackson, A.L.; Burchard, J.; Schelter, J.M.; Chau, B.N.; Cleary, M.A.; Lim, L.; Linsley, P.S. Widespread siRNA “Off-target” Transcript Silencing Mediated by Seed Region Sequence Complementarity. RNA 2006, 12, 1179-1187. [CrossRef]

22. Jackson, A.L.; Linsley, P.S. Recognizing and Avoiding siRNA Off-target Effects for Target Identification and Therapeutic Application. Nat. Rev. Drug Discov. 2010, 9, 57-67. [CrossRef] [PubMed]

23. Jackson, A.L.; Bartz, S.R.; Schelter, J.; Kobayashi, S.V.; Burchard, J.; Mao, M.; Li, B.; Cavet, G.; Linsley, P.S. Expression Profiling Reveals Off-target Gene Regulation by RNAi. Nat. Biotechnol. 2003, 21, 635-637. [CrossRef]

24. BeltCappellino, A.; Majerciak, V.; Lobanov, A.; Lack, J.; Cam, M.; Zheng, Z.-M. CRISPR/Cas9-Mediated Knockout and in situ Inversion of the ORF57 Gene from All Copies of the Kaposi's Sarcoma-Associated Her-pesvirus Genome in BCBL-1 Cells. J. Virol. 2019, 93, e00628-19. [CrossRef] [PubMed]

25. Purushothaman, P.; Dabral, P.; Gupta, N.; Sarkar, R.; Verma, S.C. KSHV Genome Replication and Maintenance. Front. Microbiol. 2016, 7, 54. [CrossRef]

26. Gilbert, L.A.; Larson, M.H.; Morsut, L.; Liu, Z.; Brar, G.A.; Torres, S.E.; Stern-Ginossar, N.; Brandman, O.; Whitehead, E.H.; Doudna, J.A.; et al. CRISPR-Mediated Modular RNA-Guided Regulation of Transcription in Eukaryotes. Cell 2013, 154, $442-451$. [CrossRef] [PubMed]

27. Gilbert, L.A.; Horlbeck, M.A.; Adamson, B.; Villalta, J.E.; Chen, Y.; Whitehead, E.H.; Guimaraes, C.; Panning, B.; Ploegh, H.L.; Bassik, M.C.; et al. Genome-Scale CRISPR-Mediated Control of Gene Repression and Activation. Cell 2014, 159, 647-661. [CrossRef] [PubMed]

28. Urrutia, R. KRAB-containing Zinc-finger Repressor Proteins. Genome Biol. 2003, 4, 231. [CrossRef]

29. Smith, J.D.; Suresh, S.; Schlecht, U.; Wu, M.; Wagih, O.; Peltz, G.; Davis, R.W.; Steinmetz, L.M.; Parts, L.; Onge, R.P. Quantitative CRISPR Interference Screens in Yeast Identify Chemical-genetic Interactions and New Rules for Guide RNA Design. Genome Biol. 2016, 17, 1-16. [CrossRef]

30. Todor, H.; Silvis, M.R.; Osadnik, H.; Gross, C.A. Bacterial CRISPR Screens for Gene Function. Curr. Opin. Microbiol. 2021, 59, 102-109. [CrossRef]

31. Hawkins, J.S.; Wong, S.; Peters, J.M.; Almeida, R.; Qi, L.S. Targeted Transcriptional Repression in Bacteria Using CRISPR Interference (CRISPRi). Methods Mol. Biol. 2015, 1311, 349-362. [CrossRef]

32. Acosta-Alvear, D.; Cho, M.Y.; Wild, T.; Buchholz, T.J.; Lerner, A.G.; Simakova, O.; Hahn, J.; Korde, N.; Landgren, O.; Kampmann, M.; et al. Paradoxical Resistance of Multiple Myeloma to Proteasome Inhibitors by Decreased Levels of 19S Pro-teasomal Subunits. Elife 2015, 4, e08153. [CrossRef]

33. Horlbeck, M.A.; Gilbert, L.A.; Villalta, J.E.; Adamson, B.; Pak, R.A.; Chen, Y.; Fields, A.P.; Park, C.Y.; Corn, J.E.; Weissman, J.S.; et al. Compact and Highly Active Next-generation Libraries for CRISPR-mediated Gene Repression and Activation. Elife 2016, 5, e19760. [CrossRef] [PubMed]

34. Wu, L.; Renne, R.; Ganem, D.; Forghani, B. Human Herpesvirus 8 Glycoprotein K8.1: Expression, Post-translational Modification and Localization Analyzed by Monoclonal Antibody. J. Clin. Virol. 2000, 17, 127-136. [CrossRef] 
35. Renne, R.; Zhong, W.; Herndier, B.; McGrath, M.; Abbey, N.; Kedes, D.; Ganem, D. Lytic Growth of Kaposi's Sarcoma-associated Herpesvirus (Human Herpesvirus 8) in Culture. Nat. Med. 1996, 2, 342-346. [CrossRef]

36. Myoung, J.; Ganem, D. Generation of a Doxycycline-inducible KSHV Producer Cell Line of Endothelial Origin: Maintenance of Tight Latency with Efficient Reactivation upon Induction. J. Virol. Methods 2011, 174, 12-21. [CrossRef]

37. Vieira, J.; O'Hearn, P.M. Use of the Red Fluorescent Protein as a Marker of Kaposi's Sarcoma-associated Herpesvirus Lytic Gene Expression. Virology 2004, 325, 225-240. [CrossRef]

38. Purushothaman, P.; Uppal, T.; Verma, S.C. Molecular Biology of KSHV Lytic Reactivation. Viruses 2015, 7, 116-153. [CrossRef] [PubMed]

39. Walter, P.; Ron, D. The Unfolded Protein Response: From Stress Pathway to Homeostatic Regulation. Science 2011, 334, 1081-1086. [CrossRef]

40. Ballestas, M.E.; Kaye, K.M. Kaposi's Sarcoma-Associated Herpesvirus Latency-Associated Nuclear Antigen 1 Mediates Episome Persistence through cis Acting Terminal Repeat (TR) Sequence and Specifically Binds TR DNA. J. Virol. 2001, 75, 3250-3258. [CrossRef] [PubMed]

41. Ballestas, M.E.; Chatis, P.A.; Kaye, K.M. Efficient Persistence of Extrachromosomal KSHV DNA Mediated by Laten-cy-Associated Nuclear Antigen. Science 1999, 284, 641-644. [CrossRef] [PubMed]

42. Hu, J.; Garber, A.C.; Renne, R. The Latency-Associated Nuclear Antigen of Kaposi's Sarcoma-Associated Herpes-virus Supports Latent DNA Replication in Dividing Cells. J. Virol. 2002, 76, 11677-11687. [CrossRef] [PubMed]

43. Schulz, T.F.; Chang, Y. KSHV Gene Expression and Regulation. In Human Herpesviruses; Cambridge University Press (CUP): Cambridge, UK, 2010; pp. 490-513.

44. Majerciak, V.; Zheng, Z.-M. KSHV ORF57, a Protein of Many Faces. Viruses 2015, 7, 604-633. [CrossRef] [PubMed]

45. Majerciak, V.; Pripuzova, N.; McCoy, J.P.; Gao, S.-J.; Zheng, Z.-M. Targeted Disruption of Kaposi's Sar-coma-Associated Herpesvirus ORF57 in the Viral Genome Is Detrimental for the Expression of ORF59, K8 $\alpha$, and K8.1 and the Production of Infectious Virus. J. Virol. 2007, 81, 1062-1071. [CrossRef]

46. Chan, S.R.; Chandran, B. Characterization of Human Herpesvirus 8 ORF59 Protein (PF-8) and Mapping of the Proces-sivity and Viral DNA Polymerase-Interacting Domains. J. Virol. 2000, 74, 10920-10929. [CrossRef] [PubMed]

47. AuCoin, D.P.; Colletti, K.S.; Cei, S.A.; Papousková, I.; Tarrant, M.; Pari, G.S. Amplification of the Kaposi's Sarcoma-associated Herpesvirus/Human Herpesvirus 8 Lytic Origin of DNA Replication is Dependent upon a Cis-acting AT-rich Region and an ORF50 Response Element and the Trans-acting Factors ORF50 (K-Rta) and K8 (K-bZIP). Virology 2004, 318, 542-555. [CrossRef] [PubMed]

48. Chen, X.; Xulin, C.; Ricciardi, R.P. Human Kaposi's Sarcoma Herpesvirus Processivity Factor-8 Functions as a Dimer in DNA Synthesis. J. Biol. Chem. 2004, 279, 28375-28386. [CrossRef]

49. Pearce, M.; Matsumura, S.; Wilson, A.C. Transcripts Encoding K12, v-FLIP, v-Cyclin, and the MicroRNA Cluster of Kaposi's Sarcoma-Associated Herpesvirus Originate from a Common Promoter. J. Virol. 2005, 79, 14457-14464. [CrossRef]

50. Hein, M.Y.; Weissman, J.S. Functional Single-cell Genomics of Human Cytomegalovirus Infection. bioRxiv $2019,775080$.

51. Radzisheuskaya, A.; Shlyueva, D.; Müller, I.; Helin, K. Optimizing sgRNA Position Markedly Improves the Efficiency of CRISPR/dCas9-mediated Transcriptional Repression. Nucleic Acids Res. 2016, 44, e141. [CrossRef]

52. Matsumura, S.; Fujita, Y.; Gomez, E.; Tanese, N.; Wilson, A.C. Activation of the Kaposi's Sarcoma-Associated Herpesvirus Major Latency Locus by the Lytic Switch Protein RTA (ORF50). J. Virol. 2005, 79, 8493-8505. [CrossRef]

53. Elbasani, E.; Falasco, F.; Gramolelli, S.; Nurminen, V.; Günther, T.; Weltner, J.; Balboa, D.; Grundhoff, A.; Otonkoski, T.; Ojala, P.M. Kaposi's Sarcoma-Associated Herpesvirus Reactivation by Targeting of a dCas9-Based Transcription Ac-tivator to the ORF50 Promoter. Viruses 2020, 12, 952. [CrossRef] 